\title{
Coronal emission lines as thermometers
}

\author{
Philip G. Judge \\ High Altitude Observatory, National Center for Atmospheric Research1, P.O. Box 3000, \\ Boulder CO 8030\%-3000,USA; judge@ucar.edu
}

\begin{abstract}
Coronal emission line intensities are commonly used to measure electron temperatures using emission measure and/or line ratio methods. In the presence of systematic errors in atomic excitation calculations and data noise, the information on underlying temperature distributions is fundamentally limited. Increasing the number of emission lines used does not necessarily improve the ability to discriminate between different kinds of temperature distributions.
\end{abstract}

Subject headings: Sun: corona

\section{Introduction}

Spectroscopic measurements of the temperature of coronal plasma have been made for decades (e.g. Seaton 1962; Noci 2003). Feldman et al. (1999); Feldman \& Landi (2008) and Landi \& Feldman (2008) presented intriguing evidence that electron temperatures of solar coronal plasma, measured from emission line spectra obtained high in the coroma, are clumped into several peaks, and are not broadly distributed. Narrow distributions of plasma temperature have important implications for the energy balance of the corona. In the methods used, frequency integrated line intensities $I$ are assumed to be simple functions $G(T)$ of the logarithm of the electron temperature, $T$, because of the dominance of two body collisional processes which lead to the well-known "coronal approximation" (e.g. Woolley \& Allen 1948; Seaton 1964). Two approaches were used. In one they solved for the differential emission measure $\xi(T)$ which is an optimal solution to the inverse problem:

$$
I_{i}=\int G_{i}(T) \xi(T) d T, \quad i=1 \ldots n
$$

\footnotetext{
${ }^{1}$ The National Center for Atmospheric Research is sponsored by the National Science Foundation
} 
where there is a set of $n$ different emission lines. The other method sought the single logarithmic temperature $T_{0}$ such that $\xi(T) \propto \delta\left(T-T_{0}\right)$, by plotting $I_{i} / G_{i}(T)$ and identifying intersection points for the observed lines. These approaches are, in fact, formally equivalent (McIntosh et al. 1998). In both cases, information must be added to obtain both $\xi(T)$ and

$T_{0}$ : in the first case the problem has to be "regularized" as it is ill-posed (e.g. Craig \& Brown 1986), one searches for example for the "least structured" solution $\xi(T)$ that is compatible with the data. In the second case it is assumed that the plasma is indeed approximately isothermal. In the analysis of Feldman \& Landi (2008), the individual peaks in the $\xi(T)$ functions have widths of 0.1-0.2 in $T$, and the curves of $I_{i} / G_{i}(T)$ intersect one another within similar margins.

Some general questions arise. Given typical uncertainties in observable and model parameters, are the observed data compatible with different $\xi(T)$ distributions? What, then, is the accuracy of the derived temperatures? In this paper these questions are addressed by asking, how broad can $\xi(T)$ functions be to be incompatible with observed data. By how much can one change the temperature of an isothermal plasma before the differences in line intensities become significant? In the problem at hand there are unavoidably large and systematic uncertainties in the $G(T)$ functions. These uncertainties limit the information which can be extracted from emission line spectra. It is found that acceptable widths $w$ of the $\xi(T)$ functions exceed the precision by which the lines can in principle determine that two different plasmas have slightly different temperatures. Thus, these widths set the lower limit to the ability of emission line techniques to diagnose electron temperatures.

\section{Calculations of temperature sensitive lines}

\subsection{Uncertainties in $G(T)$}

$G(T)$ functions depend linearly on electron impact excitation rates, on elemental abundances, and on factors influencing the ionization fraction for a given line. For coronal ions, uncertainties in electron impact excitation cross sections, based primarily upon sophisticated calculations, are typically $\pm 10 \%$ but can be considerably higher (e.g. Storey et al. 2002). Other uncertainties in $G(T)$ arise from element abundance uncertainties, boundfree cross sections (ionization, recombination), and can include systematic errors from nonionization equilibrium effects, from any significant non-thermal populations of electrons, modifications of dielectronic recombination rates due to finite density plasmas (e.g. Summers 1974; Badnell et al. 2003; Judge 2007), and even radiative transfer. Given these considerations, we adopt fractional uncertainties of $\epsilon=0.2$ for all $G(T)$ functions, which should probably be considered a lower limit. 


\subsection{Numerical calculations}

Calculations were performed for lines of Fe VIII - Fe XVII using the DIPER package (Judge 2007). The upper left panel of Figure1 1 shows $G(T)$ functions computed for a typical

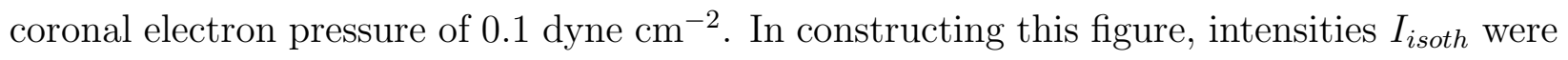
computed using an isothermal plasma at $1.3 \times 10^{6} \mathrm{~K}$. Intensities were then recomputed using a Gaussian function with unit $(-\infty \rightarrow+\infty)$ integral:

$$
\xi(T)=\frac{1}{\sqrt{\pi} w} e^{-\left(\frac{T-T_{0}}{w}\right)^{2}}
$$

The intensities were recomputed, and a value of $\chi^{2}$ computed using the above uncertainties in $G(T)$ added in quadrature to the uncertainty from photon counting statistics, assuming that the brightest line in the spectrum has accumulated $10^{4}$ counts. These uncertainties are not unrealistic values for what might be expected from UV and EUV spectrographs of the modern era. This particular calculation shows that an isothermal plasma with $T=T_{0}$ cannot formally be distinguished from a plasma with Gaussian distributions of width $w$ less than 0.1. Informally, the figure shows a systematic increase in the ratio $I / I_{i s o t h}$ in lines with $G(T)$ peaking away from $T_{0}$. One may be tempted to claim that the curvature indicates the presence of broader distributions. But these calculations are made with the same set of $G(T)$ functions, where in reality one would compare these theoretical values with those (unknown) functions, free of systematic errors, present in the real Sun.

\subsection{Analytical approximations}

To generalize these results, analytical results can be obtained using suitably simple functions to approximate $G_{i}(T)$. Here, Gaussian functions are adopted both for $\xi(T)$, as in eq. (2), and $G_{i}(T)$ :

$$
G_{i}(T)=a_{i} e^{-\left(\frac{T-T_{i}}{v_{i}}\right)^{2}}
$$

Very similar results are obtained using $\xi(T)$ represented by a "top-hat" function of full width $2 w$. These were chosen because they are not qualitatively dissimilar from the shapes of typical lines, and they are analytically simple. Then

$$
\begin{aligned}
I_{i} & =\frac{a_{i}}{\sqrt{\pi} w} \int_{-\infty}^{\infty} e^{-\left(\frac{T-T_{i}}{v_{i}}\right)^{2}} e^{-\left(\frac{T-T_{0}}{w}\right)^{2}} d T, \quad i=1 \ldots n \\
& =\frac{a_{i} v_{i}}{\sqrt{w^{2}+v_{i}^{2}}} e^{-\frac{\left(T_{0}-T_{i}\right)^{2}}{w^{2}+v_{i}^{2}}}
\end{aligned}
$$


When $w \rightarrow 0$, then $I_{i} \equiv I_{i}^{0}=a_{i} e^{-\left(\frac{T_{0}-T_{i}}{v_{i}}\right)^{2}}$, i.e. the isothermal case. If $w^{2} \ll v_{i}^{2}$ and for source temperatures $T_{1}=T_{0}+\tau\left(\tau \ll\left|T_{0}-T_{i}\right|\right.$ - large values of $\left|T_{0}-T_{i}\right|$ are needed for significant temperature sensitivity between at least some lines), these expressions can be expanded as a Taylor series, to yield changes in $I_{I}$ as functions of small values of $w$ and $\delta$. The first derivatives are

$$
\begin{aligned}
\frac{\partial I_{i}}{\partial T_{0}} & =2 I_{i}^{0} \frac{T_{i}-T_{0}}{w^{2}+v_{i}^{2}} \\
\frac{\partial I_{i}}{\partial w} & =2 I_{i}^{0} w \frac{1}{w^{2}+v_{i}^{2}}\left(\frac{\left(T_{i}-T_{0}\right)^{2}}{w^{2}+v_{i}^{2}}-\frac{1}{2}\right) .
\end{aligned}
$$

Dividing by $I_{i}$, the first order fractional changes in $I_{i}$ resulting from small changes $\tau$ and $w$ are:

$$
\begin{aligned}
& \Delta_{T}=\frac{1}{I_{i}^{0}} \frac{\partial I_{i}}{\partial T_{0}} \tau \approx 2 \frac{T_{i}-T_{0}}{v_{i}^{2}} \tau \\
& \Delta_{w}=\frac{1}{I_{i}^{0}} \frac{\partial I_{i}}{\partial w} w \approx \frac{2 w^{2}}{v_{i}^{2}}\left(\frac{\left(T_{i}-T_{0}\right)^{2}}{v_{i}^{2}}-\frac{1}{2}\right)
\end{aligned}
$$

These correction terms can be used to compute the reduced $\chi^{2}$ statistic, to first order, using the uncertainties $\epsilon_{i}$ :

$$
\chi^{2}=\frac{1}{n-1} \sum_{i}^{n} \frac{\left(I_{i}-I_{i}^{0}\right)^{2}}{I_{i}^{0} \epsilon_{i}^{2}}=\frac{1}{n-1} \sum_{i}^{n} \frac{\Delta_{i}^{2}}{\epsilon_{i}^{2}}
$$

Define $x_{i}=\left|T_{0}-T_{i}\right| / v_{i}$, which measures the difference in temperature of the peak of the source relative to the peak of line $i$ in units of the Gaussian width of $\left.G_{(} T\right)$. Any sensible set of lines must include values of $x_{i}$ varying from 0 to numbers $\gg 1$. But for $x_{i} \gtrsim 3$, the lines become very weak with accompanying large observational uncertainties. Practically speaking, we can consider most values of $x_{i}$ to be spread between 0 and 3 . Any given set of lines cannot be used to discriminate an isothermal source at temperature $T_{0}$ from another at temperature $T_{0} \pm \tau$ when (using $\Delta=\Delta_{T}$ ), the majority of lines satisfy

$$
|\tau| \lesssim \frac{\epsilon_{i}}{2 x_{i}} v_{i}, \forall i=1 \ldots n
$$

Similarly one cannot discriminate an isothermal source at temperature $T_{0}$ from a broadened distribution with Gaussian parameter $\tau$ when (using $\Delta=\Delta_{w}$ )

$$
w \lesssim \sqrt{\frac{\epsilon_{i}}{\left|2 x_{i}^{2}-1\right|}} v_{i}, \forall i=1 \ldots n
$$

Using $x_{i} \sim 1, v_{i} \sim 0.15, \epsilon \sim 0.2$, the set of emission lines lines has sensitivities to the peak of the temperature and its width given by $w$ of

$$
\tau \sim 0.015, \quad w \sim 0.13
$$


This result for $w$ is in reasonable agreement with the numerical calculation. The "bluntness of the thermometer" is, in this approximation, measured by $w$. This quantity therefore determines the accuracy of temperature measurements. The accuracy is a factor of 8 worse than the precision with which different isothermal plasma temperatures can be discerned, relative to one another, which is represented by $\tau$. The width $w$ is weakly dependent on the intrinsic uncertainties $\epsilon_{i}$, linearly dependent on widths $v_{i}$ of the $G_{i}(T)$ function, and inversely proportional to $\left|x_{i}\right|$.

Given the weakness of lines with large $x_{i}$ values, the nature of the uncertainties $\epsilon_{i}$, it relatively little can be done to increase the thermometer's accuracy. However, one can try to include very strong lines (large $a_{i}$ ) which are formed far from their peak temperature $T_{i}$, i.e. when $x_{i}>3$, say, such that the counts are still sufficiently large to make $\epsilon_{i}$ approach the systematic uncertainties in atomic calculations. One example might be the strong line of Fe IX near $17.1 \mathrm{~nm}$ whose $G(T)$ function is prominent in Figure 1. In such cases

$$
w \propto v_{i} \sqrt{\epsilon_{i}} / x_{i} \quad\left(x_{i} \gg 1\right)
$$

Note however, that such lines tend also to have large widths $v_{i}$.

Temperature estimates cannot however be improved simply by analyzing more lines with similar values of $x_{i}$, because then the largest acceptable width $w$ is almost independent of the number of lines $n$ used. The uncertainties in $\epsilon_{i}$ are, in an important sense, irreducible, as, even in the limit of perfect observations, they result from uncertainties in atomic cross sections and in our lack of knowledge of the conditions controlling level populations in the solar coronal plasmas.

\section{Discussion}

The largest values of $w$ compatible with observations yield the accuracy with which emission lines can measure electron temperatures. The widths of the distributions compatible with known sources of uncertainties set a natural limit on the sharpness of a detectable peak in the underlying emission measure distributions. Typically the full width, $2 w$, is 0.2 to 0.3 in the logarithmic electron temperature. The precision is an order of magnitude better, being estimated using the sensitivity of the spectra to two isothermal plasmas differing in logarithmic temperature by $\tau$. Although this implies that two strictly isothermal plasmas could be differentiated by using line ratios to a precision of order $\epsilon_{i} v_{i} / 2 \sim 0.015$ in the logarithmic electron temperature, we will perhaps never know if such isothermal plasmas exist with a width narrower than $w$, based upon these data.

These properties prompt the following comments: 
1. Error bars quoted below $\sim 0.1$ in logarithmic temperatures are not credible using these techniques. Landi and Feldman quote error bars of 0.04 and 0.05 in their work which seeks to provide spectroscopic evidence that the coronal temperature is "quantized".

2. The widths (FWHM) of peaks in the $\xi(T)$ functions found by Landi \& Feldman (2008, their figure 5) are between 0.1 and 0.2 . These widths are characteristic of the limit with which emission lines can determine the isothermality of the emitting plasma, and are therefore determined more by regularization than by the data themselves. Their analysis is consistent with isothermal plasma, but is limited by the uncertainties discussed here.

3. Coronal temperatures are controlled by (unknown) heating mechanisms and cooling by heat conduction, flows, radiation. The scalings of Rosner et al. (1978), based on simple energy balance considerations, show that a given coronal electron temperature $T_{e}$ in a loop of length $L$ requires an energy flux density

$$
\mathcal{F} \propto\left(T_{e} L\right)^{7 / 2}
$$

to sustain it agains conductive and radiation losses. Therefore, for a given $L$, if $T_{e}$ is uncertain to \pm 0.15 in its logarithm, the absolute value of $\log _{10} \mathcal{F}$ can be determined from a given length loop to an accuracy of $\sim \pm 0.5$. The energy flux is constrained only to somewhere within a range spanning a factor of ten using emission lines. Conversely, variations of the energy flux $\mathcal{F}$ dissipated in coronal loops by $\lesssim 0.5$ in the logarithm cannot be distinguished through temperature dependent emission line methods.

4. Relative changes in $\mathcal{F}$ could, however, be detected to within about $\pm 12 \%$, but again this precision depends on the plasma being nearly isothermal (i.e. actual $w \sim \tau$ ), which is neither known from the data nor expected from first principles.

The analytical results offer some hope that carefully selected sets of emission lines can be used to reduce the widths of distributions compatible with the data. Specifically, using lines within the same element removes errors arising from incorrect abundances, and can include lines with large values of $\left|x_{i}\right|$. In this case just a few lines with different values of $x_{i}$ can reduce $w$ via equation (14)). Using lines from within the same ion stage yields significantly smaller values of $\epsilon_{i} \sim 0.1$, but while the precision improves linearly with $\epsilon_{i}$ the accuracy improves only quadratically.

The author is grateful to Scott McIntosh for carefully reading the manuscript. 


\section{REFERENCES}

Badnell N. R., O’Mullane M. G., Summers H. P., Altun Z., Bautista M. A., Colgan J., Gorczyca T. W., Mitnik D. M., Pindzola M. S., Zatsarinny O., 2003, A\&A 406, 1151

Craig I. J. D., Brown J. C., 1986, Inverse problems in astronomy, Hilger, Bristol

Feldman U., Doschek G. A., Schühle U., Wilhelm K., 1999, ApJ 518, 500

Feldman U., Landi E., 2008, Physics of Plasmas 15(5), 56501

Judge P., 2007, THE HAO SPECTRAL DIAGNOSTIC PACKAGE FOR EMITTED RADIATION (haos-diper) Reference Guide (Version 1.0), Technical Report NCAR/TN473-STR, National Center for Atmospheric Research

Landi E., Feldman U., 2008, ApJ 672, 674

McIntosh S., Brown J. C., Judge P. G., 1998, A\&A 333, 333

Noci G., 2003, Memorie della Societa Astronomica Italiana 74, 704

Rosner R., Tucker W. H., Vaiana G. S., 1978, ApJ 220, 643

Seaton M. J., 1962, Observatory 928, 111

Seaton M. J., 1964, Planet. Space Sci. 12, 55

Storey P. J., Zeippen C. J., Le Dourneuf M., 2002, A\&A 394, 753

Summers H. P., 1974, MNRAS 169, 663

Woolley R. D. V. R., Allen C. W., 1948, MNRAS 108, 292 

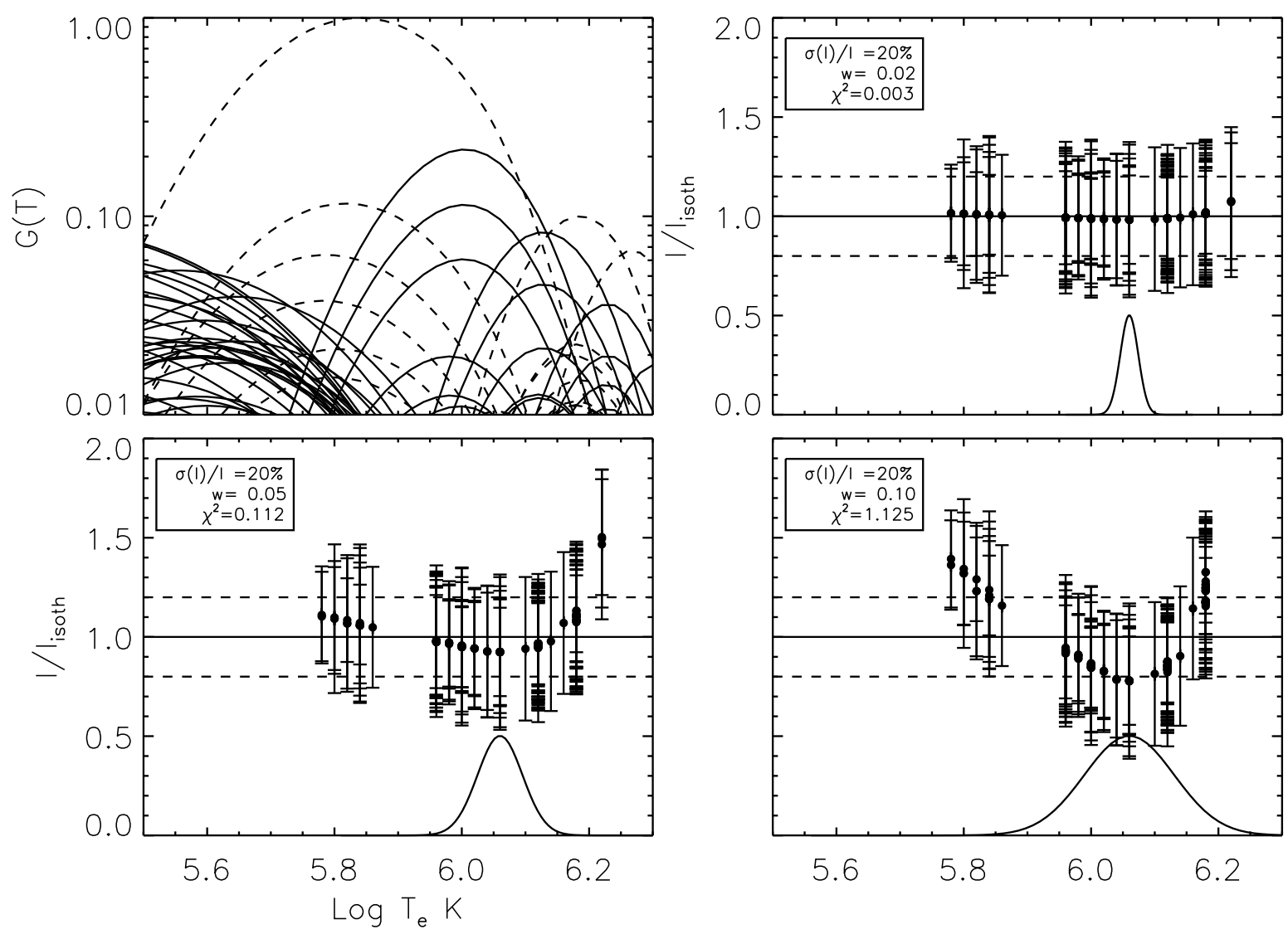

Fig. 1.- $G(T)$ functions for Fe VIII-Fe XVII are shown as a function of $T$ (logarithm of the electron temperature) in the upper left panel. Solid and dashed lines mark ions with odd and even charges repectively, for clarity. The other panels plot the ratios of intensities computed using the Gaussian functions shown (solid lines) divided by the intensities for an isothermal calculation centered at the same temperature (in this case $T=\log _{10} 1.2 \times 10^{6}$ $\mathrm{K})$. The uncertainties are set to $\gtrsim 20 \%$ of the computed line intensities (see text) and the computed $\chi^{2}$ parameters are listed with the Gaussian width $w$. 